\title{
Schaden Antihypertensiva älteren Patienten?
}

\author{
Bei älteren Hypertonikern besteht \\ häufig die Sorge, durch eine Blut- \\ drucksenkung möglicherweise or- \\ thostatische Regulationsstörungen \\ auszulösen und damit das Sturzrisiko \\ zu erhöhen. In der PARTAGE-Studie \\ bestätigten sich diese Probleme \\ nicht.
}

- An 994 Bewohnern von Altenheimen (im Mittel $88 \pm 5$ Jahre) wurde untersucht, welche Auswirkung die antihypertensive Therapie hinsichtlich einer orthostatischen Hypotonie hat.

Eine orthostatische Hypotonie wurde diagnostiziert, wenn eine oder drei Minuten nach dem Aufstehen der systolische Blutdruck um mindestens 20 $\mathrm{mmHg}$ und/oder der diastolische Blutdruck um mindestens $10 \mathrm{mmHg}$ abnahm.

Antihypertensiv behandelte und unbehandelte Probanden hatten keine signifikant unterschiedliche Häufigkeit der orthostatischen Hypotonie (18\% vs.

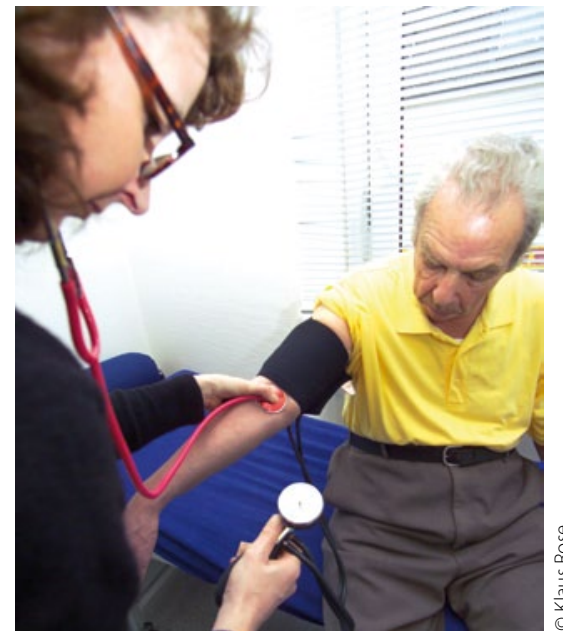

Blutdrucksenkung bei Hochbetagten: kaum orthostatische Hypotonie.

17\%). Besonders bemerkenswert war, dass ausreichend antihypertensiv behandelte Patienten signifikant seltener eine orthostatische Hypotonie aufwiesen als diejenigen, deren Blutdruck nicht zufriedenstellend eingestellt war.

\section{Kommentar}

Das wichtigste Ergebnis dieser Studie ist, dass die Hypertonietherapie bei älteren Patienten nicht mit einem erhöhten Risiko einer orthostatischen Hypotonie verbunden ist. Deswegen ist auch ein mögliches Sturzrisiko wegen einer orthostatischen Dysregulation kein Argument, ältere Patienten nicht oder nicht ausreichend antihypertensiv zu behandeln.

Das Ergebnis ist an einer repräsentativen Anzahl von Patienten erhoben worden und hat wichtige Auswirkungen auf die Praxis. Noch immer wird therapeutische Zurückhaltung geübt mit der Begründung, eine ausreichende Blutdruckeinstellung könnte orthostatische Probleme verursachen. Dies ist den Ergebnissen der PARTAGE-Studie zufolge nicht berechtigt.

W. ZIDEK =

\footnotetext{
- F. Valbusa et al.

Orthostatic hypotension in very old individuals living in nursing homes: the PARTAGE study. J. Hypertension 2012; 30: 53-60
}

\section{Mit dem Bleistift aufgespießt}

\section{Eine 75-jährige Frau suchte wegen einer bläulichen Verfärbung von etwa $1 \mathrm{~cm}$ Durchmesser an der Streckseite des Zeigefingerend- glieds den Arzt auf. Die Patientin konnte sich noch daran erinnern, dass sie sich vor 50 Jahren an dieser Stelle mit einem spitzen Bleistift gestochen hatte.}

- Damals war die Spitze des Bleistifts abgebrochen und blieb unter der Haut stecken. In den folgenden Jahren war eine leichte Erhabenheit entstanden, die sich dunkel verfärbte und allmählich druckschmerzhaft wurde (Abb. A, Pfeil). Der histologische Befund einer Biopsie dieser Stelle zeigte eine Gewebedegeneration mit Kalzifikationen und den Befund eines Granuloms mit $\begin{aligned} & \text { Das Granulom (A) } \\ & \text { und der histolo- } \\ & \text { gische Befund (B). }\end{aligned}$
Histiozyten, die mit
schwarzem Graphit
belad en waren
(Abb. B). Aufgrund
der Anamnese, des
klinischen Befundes und der Histologie wurde die Diagnose eines Bleistiftminen-Granuloms gestellt.

\section{Kommentar}

Bleistiftminen bestehen aus einer Verbindung von Graphit, Lehm, Wachs und Lack. Jede dieser Komponenten kann eine granulomatöse Reaktion der Haut erzeu-
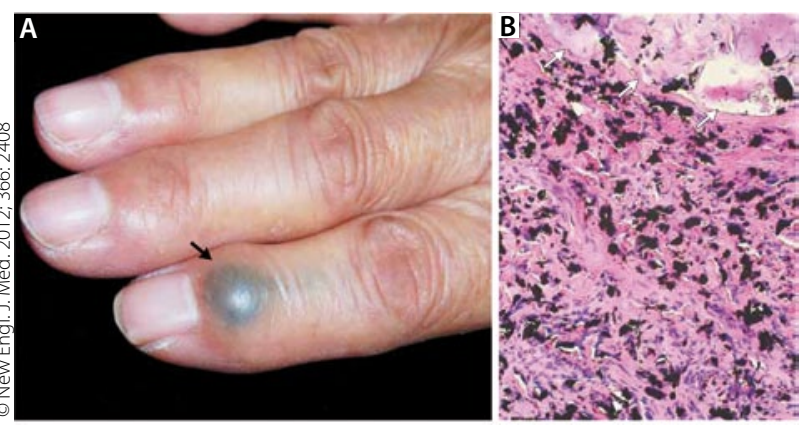

gen. Auf Wunsch der Patientin wurde das Bleistiftminen-Granulom nicht entfernt. Auch nach zehn Monaten unter klinischer Beobachtung hatte sich der Befund nicht geändert.

H. S. FÜESSL =

- T. Tagucki und Y. Terada

(Kochi Medical School, Kochi, Japan): PencilCore Granuloma. New Engl. J. Med. 2012; 366: 2408 